\title{
Philosophiques
}

\section{Voltaire et l'Affaire Calas au théâtre : une vraie cause au service des mythologies révolutionnaires}

\section{Michèle Sajous D’Oria}

Volume 21, numéro 1, printemps 1994

URI : https://id.erudit.org/iderudit/027252ar

DOI : https://doi.org/10.7202/027252ar

Aller au sommaire du numéro

\section{Éditeur(s)}

Société de philosophie du Québec

\section{ISSN}

0316-2923 (imprimé)

1492-1391 (numérique)

Découvrir la revue

\section{Citer cet article}

Sajous D’Oria, M. (1994). Voltaire et l'Affaire Calas au théâtre : une vraie cause au service des mythologies révolutionnaires. Philosophiques, 21(1), 107-123. https://doi.org/10.7202/027252ar

\section{Résumé de l'article}

Le théâtre, au lendemain même de la prise de la Bastille, s'était affirmé comme tribune révolutionnaire et " école du citoyen ". La décision, de la part des assemblées révolutionnaires, de transporter les cendres de Voltaire au Panthéon, ne pouvait manquer d'être une occasion pour célébrer le philosophe au théâtre et cinq pièces, toutes sur l'Affaire Calas, furent représentées entre le 17 décembre 1790 et le 31 juillet 1791 . Les cinq auteurs centrent leur action sur le drame familial et sur le climat de fanatisme religieux dans lequel elle s'était déroulée. Deux mots, fanatisme et fanatique, représentent la pierre de touche et font le passage entre l'époque réelle de l'Affaire Calas (1761-1762) et l'époque de sa représentation théâtrale (1790-1791). Ils recouvrent l'événement et son moment historique précis, ils constituent une notion pour laquelle les philosophes s'étaient battus et prennent la valeur d'un symbole paradigmatique que la Révolution prétendait achevé. Les pièces s'articulent selon un continuel renvoi entre le temps réel et le temps de la représentation : le passé - le temps du fanatisme et du despotisme - annonce l'avenir l'anéantissement de l'un et de l'autre - qui est le présent des spectateurs. 


\title{
VOL TAIRE ET L'AFFAIRE CALAS AU THÉÁTRE : UNE VRAIE CALSE AU SERVICE DES MYTHOLOGIES REVOLUTIONAIRES
}

\author{
par \\ Michèle Sajous D'Oria
}

RÉSUMÉ : Le thêâtre, au lendemain même de la prise de la Bastille, s'était affirmé comme tribune révolutionnaire et "école du citoyen ». La décision, de la part des assemblées révolutionnaires, de transporter les cendres de Voltaire au Panthéon, ne pouvait manquer d'être une occasion pour célébrer le philosophe au théâtre et cinq pièces, toutes sur l'Affaire Calas, furent représentées entre le 17 décembre 1790 et le 31 juillet 1791. Les cinq auteurs centrent leur action sur le drame familial et sur le climat de fanatisme religieux dans lequel elle s'était déroulée.

Deux mots, fanatisme et fanatique, représentent la pierre de touche et font le passage entre l'époque réelle de l'Affaire Calas (1761-1762) et l'époque de sa représentation théâtrale (1790-1791). Ils recouvrent l'événement et son moment historique précis, ils constituent une notion pour laquelle les philosophes s'étaient battus et prennent la valeur d'un symbole paradigmatique que la Révolution prétendait achevé. Les pièces s'articulent selon un continuel renvoi entre le temps réel et le temps de la représentation: le passé - le temps du fanatisme et $d u$ despotisme - annonce l'avenir - l'anéantissement de l'un et de l'autre - qui est le présent des spectateurs.

ABSTRACT :The morning after the fall of the Bastille, theatre imposed itself as both a revolutionary pulpit and an " école du citoyen " (a school for the citizen). The revolutionary assemblies' decision to transfer Voltaire's ashes to the Panthéon was the occasion to celebrate the philosopher at the theatre: five plays, all regarding the Affaire Calas, where offered to the public between 17 December 1790 and 31 July 1791. All five authors based their plays on both the family drama and on the climate of religious fanaticism in which the former occurred.

Fanaticism and fanatic are the words which act as the touchtone and which allow the transition from the 
historical period which is that of the Affaire Calas (17611762) to its presentation on the stage (1790-1791). The plays acquire structure through constant cross-reference opposing the historical period and the time of the dramatic representation: the past, a time of fanaticism and despotism, foreshadows the abolishment of both in the future; that is, the spectator's present.

«Vingt-cinq ans n'ont pas affaibli le sentiment de l'exécrable injustice qui a fait périr Calas sur l'échafaud. Cet événement a paru susceptible d'être mis sur la scène au moment où le thêâtre a pu jouir de la Liberté », pouvait-on lire dans « Le Moniteur » du 29 décembre $1790^{\mathrm{I}}$.

Le théâtre, au lendemain même de la prise de la Bastille, après un siècle de privilèges et de censure, s'était affirmé immédiatement comme tribune révolutionnaire. Il s'était fait lieu public de communication en suivant au plus près une actualité riche en événements et en célébrant les héros du jour et il s'était voulu « école du citoyen ». C'est ainsi qu'une floraison d'œuvres de circonstances et « civiques » furent récitées sur les scènes révolutionnaires et offrirent quotidiennement à un auditoire nombreux et attentif la représentation d'une Révolution régénératrice ${ }^{2}$.

La décision, de la part des assemblées révolutionnaires, de transporter les cendres de Voltaire au Panthéon, ne pouvait manquer d'être une occasion pour célébrer le philosophe au thêâtre. Cinq pièces entre le i7 décembre i79o et le 3r juillet i79I vont rendre hommage au grand homme : Calas, ou le fanatisme, drame de Lemierre d'Argy représenté le I7 décembre I790 au Thêâtre du Palais Royal; Jean Calas, tragédie de Laya, le I 8 décembre 1790 au théâtre de la Nation; La bienfaisance de

I. Compte rendu dans J.L. Laya, Jean Calas, Avignon, Garrigan, I79I, voir aussi J.L. Laya, Jean Calas, Paris, Maradan et Perlet, r79I (précédée d'une Préface historique sur Jean Calas et suivie d'un nouveau $V^{e}$ acte. $C f$. aussi la Préface du texte imprimé de Ia Veuve Calas à Paris, ou le triomphe de Voltaire, Paris, 179I, de Pujoulx : « Je trouvai digne d'un Auteur dramatique, qui sent toute l'influence des représentations théâtrales sur les mœurs du peuple, de rendre ainsi successivement un hommage public aux deux hommes (Pujoulx avait aussi dédié une pièce à la mort de Mirabeau) à qui la patrie reconnaissante a décerné le titre de grands et les honneurs du triomphe. » Willemain d' Abancourt consacra aussi une pièce à l'exhumation de Voltaire à Romilly le 9 mai I79ı : Voltaire à Romilly jouée au thêâtre Molière le so juillet $\mathbf{1 7 9 I}$ (publiée chez Brunet, la même année dans La bienfaisance de Voltaire).

2. Par exemple : La France régénérée, Nicodème dans la Lune ou la Révolution pacifique, Le réveil d’Épiménide. Pour Nicodème dans la Lune, v. notre édition critique, Schena-Nizet. I983. 
Voltaire, pièce dramatique en un acte de Willemain d'Abancourt, le $3^{1}$ mai I79ı au Thêâtre de la Nation; Jean Calas, tragédie en cinq actes de M.-J. Chénier, représentée le 6 juillet I7gr au Thêâtre de la Nation et Ia Veuve Calas à Paris, ou le triomphe de Voltaire, pièce en un acte de Pujoulx, au théâtre des Italiens le 3I juillet ${ }^{3}$. L'unanimité du choix du sujet, de la part de cinq auteurs differents, fait penser que l'Affaire avait conservé une forte résonnance publique et symbolique, qui était d'ailleurs confirmée par la présence du nom de Calas sur le sarcophage qui transportait les cendres du philosophe ${ }^{4}$. Elle correspond en fait à l'image révolutionnaire de Voltaire la plus véhiculée, «l'homme aux Calas », mème si la panthéonisation, pour les révolutionnaires qui l'avaient décrétée, avait une signification bien plus profonde, c'est-à-dire reconnaître en Voltaire, comme plus tard en Rousseau (le I2 octobre 1793$)^{5}$, le véritable précurseur de la Révolution.

D'autre part, d'un point de vue plus strictement dramaturgique l'affaire Calas est un bon sujet de drame et répond parfaitement au fondement « philosophique » du genre : l'histoire d'un simple bourgeois et de sa famille sur lesquels pèsent des menaces et qui se trouvent confrontés à des situations de héros de tragédie. « Mais si notre cœur entre pour quelque chose dans l'intérêt que nous prenons aux personnages de la Tragédie, c'est moins parce qu'ils sont héros ou Rois que parce qu'ils sont hommes et malheureux $»$, affirmait Beaumarchais dans son Essai sur le genre dramatique sérieux. C'était d'ailleurs le sujet idéal que souhaitait aussi, en I77I, L. S. Mercier, dans son roman utopique L'an deux mille quatre cent quarante , quand la Révolution - imaginaire - aurait eu lieu :

[...] On leva la toile. La scène était à Toulouse. Je vis son capitole, ses capitouls, ses juges, ses bourreaux, son peuple fanatique. La famille

3. Cf. Lemierre d'Argy, Calas, ou le fanatisme, Paris, au bureau des Rêvolutions de Paris, I79I; J.L. Laya, Jean Calas, op. cit;; Willemain d'Abancourt, La bienfaisance de Voltaire, op. cit; M.J. Chènier, Jean Calas, Paris, Moutard, 1793; et Pujoulx, La veuve de Calas à Paris, op.cit.

4. Sur les parois du sarcophage on pouvait lire : $\mathrm{Il}$ vengea Calas, Sirven, La Barre et Montbailly. Poète, philosophe, historien, il a fait prendre un grand essor à l'esprit humain, et nous a préparé à devenir libres. Il combattit les athées et les fanatiques. Il inspira la tolérance. Il réclama les droits de l'homme contre la servitude de la féodalité » (cf. le « Mercure de France » du zo juillet 1791).

5. Il est à remarquer que les pièces sur Rousseau concerne davantage des épisodes de sa vie : son enfance, sa mort, (J-N. Bouilly, Jean-Jacques Rousseau à ses demiers moments (179o); Anonyme, J.J. Rousseau dans l'ile de Saint-Pierre (1791); J. Aude, Jean-Jacques Rousseau au Paraclet (1793); G.-S. Andrieux, L'enfance de Jean-Jacques Rousseau (1794). 
de l'infortuné Calas parut et m'arracha des larmes. I... Ce qui m’attendrit, c'était la vérité qui respirait dans ce drame. On s'était bien gardé de défigurer ce sujet touchant par l'invraisemblance et la monotonie de nos vers rimés. Le poète avait suivi la marche de cet événement cruel; et son âme ne s'était attachée qu'à saisir ce que la situation déplorable de chaque victime faisait naître, ou plutôt il empruntait leur langage l...l. A la fin de cette tragédie on me montrait au doigt, et l'on disait : « voilà le contemporain de ce siècle malheureux. Il a entendu le cri de cette populace effrénée que soulevait ce David; il a été témoin des fureurs de ce fanatisme absurde !» Alors je m'enveloppai de mon manteau, je me cachai le visage, et je rougis pour mon siècle ${ }^{6}$.

Comme l'aurait voulu Mercier, nos cinq auteurs centrent leur action sur le drame familial et sur le climat de fanatisme religieux dans lequel l'affaire s'est déroulée ${ }^{7}$. Mais si Mercier pouvait avoir une bonne lecture du contexte historique et politique du temps de l' Affaire, et imaginer le scandale qu'elle pouvait susciter dans l'avenir, il lui échappait, malgré sa Révolution-fiction, la dimension dialectique qui devait par contre sous-tendre les cinq drames révolutionnaires.

En fait ce sont deux mots, largement diffusés à l'époque de l'Affaire, fanatisme et fanatique, qui représentent la pierre de touche et font le passage entre l'époque réelle de l'Affaire Calas ( $\left.1761^{-}-1762\right)$ et l'époque de sa représentation théâtrale (r790-i79I). Ils recouvrent l'événement et son moment historique précis, ils constituent, sur le plan conceptuel, une notion pour laquelle les philosophes, et en particulier Voltaire, se sont battus (bien avant et bien après l' Affaire), et enfin, ils prennent la valeur d'un symbole paradigmatique que la Révolution prétend achevé. C'est ainsi que les pièces s'articulent selon un continuel renvoi entre le temps réel et le temps de la représentation : le passéle temps du fanatisme et du despotisme - annonce l'avenir - l'anéantissement de l'un et de l'autre - qui est le présent des spectateurs. Le procédé pourrait s'apparenter à celui des récits utopiques, mais la

6. L-S. Mercier, L'an deux mille quatre cent quarante, Ière partie, chap. XXVII, Salle de spectacles, (première édition 1771), Genève, slatkine (reprod. èd. an VII), 1979. Mercier avait aussi écrit en 1765 un poème intitulé Calas sur l'échafaud à ses juges, en 7 pages et en I774 un drame en prose Ie Juge.

7. Le fanatisme religieux avait déjà été traité au théâtre avec Mahomet de Voltaire (174I), la Veuve du Malabar de Lemierre (177o), Des druides de Le Blanc de Guillet (I772). Mais les pièces sur les protestants étaient interdites avant 1789: par exemple I'Honnête criminel de Fenouillot de Falbaire (imprimée en 1767) fut représentée pour la première fois en janvier I790, et pourtant la pièce, selon Grimm (Correspondance, 1767 ) manquait de « philosophie », c'est-à-dire n'affrontait pas vraiment la question des protestants. 
Révolution n'est pas une utopie; elle est une réalité, un «nouvel ordre de choses », immédiatement perceptible. C'est en fait le passé qui perd de sa réalité et devient un monde, « ancien », où tout était possible, en négatif, où coexistaient fanatisme et despotisme, et qui a disparu.

A ce point, il faut replacer l'Affaire Calas dans son contexte, non plus « tableau » dramatique et « utopie » révolutionnaire, mais réel, pour tenter de comprendre comment en $176 \mathrm{I}$, malgré une évolution certaine des mentalités, on pouvait mourir d'intolérance et de fanatisme. Toulouse, à l'époque de l'affaire, comptait environ 50.000 catholiques et 200 calvinistes. Le rapport numérique était tel que les protestants ne pouvaient avoir un rôle collectif significatif dans la société toulousaine; d'autre part les protestants étaient soumis à des lois sévères appliquées avec plus ou moins de rigueur par le Parlement de Toulouse. Dans ce contexte, les rapports entre catholiques et protestants ne posaient apparemment pas de difficultés particulières, du moins en ce qui concernait la sphère des rapports privés. Le comportement des catholiques envers les protestants et l'application relativement tolérante des lois (catholiques), pourraient être qualifiés de tolérance par indifférence. Et pourtant un drame privé, le suicide camouflé (ou, selon la thèse de l'accusation, l'assassinat) d'un jeune homme appartenant à une famille aisée de commerçants protestants, déclencha un phénomène de fanatisme collectif, qui aboutit à la condamnation du père du jeune homme.

Le passage de la tolèrance par indifférence (comportement individuel) à l'intolérance active (comportement collectif) ne peut s'expliquer que par une hostilité latente - due, comme on le sait, à des raisons historiques et à des facteurs politiques, économiques et sociaux contingents $^{8}$ - envers la communauté protestante qui est rejetée en tant que groupe et contre laquelle les frustrations, le besoin de bouc émissaire peuvent se déchaîner. La tolérance par indifférence est en fait une des formes masquées de l'intolérance et ouvre la voie au fanatisme. C'est bien ainsi que l'entendait Voltaire quand il érigeait la tolérance en principe dont l'écho tardif fut l'Édit de I787. Mais l'Édit fut vite dépassé par les événements au point que la Révolution récusa le mot de tolérance comme une expression «dominatrice » de l'ancien régime

8. Parmi les facteurs politiques, économiques et sociaux, citons la guerre de sept ans et ses conséquences économiques; l'exclusion de certaines professions (offices publics, professions juridiques et médicales) pour lesquelles on exigeait un certificat de catholicité; cf. D. Bien, L'Affaire Calas, Toulouse, Eché, 1987. 
qui ne devait plus faire partie du « langage d'un peuple éclairé »9. D’autre part, la « tolérance » envers les protestants n'avait-elle pas été pour Voltaire une étape ou un moyen et son intervention en faveur de la famille Calas une occasion d'écraser l'Infâme ${ }^{\mathrm{IO}}$ ? Il ne faut donc pas s'étonner si, dans les pièces sur l'Affaire, la question protestante n'est que marginale, ou référée à l'histoire dont les seuls protagonistes sont les rois. L'éclatant précédent thêatral sur la question protestante avait été le Charles IX de Chénier, encore une fois une pièce qui se voulait didactique pour les rois ${ }^{11}$. De même les allusions aux guerres de Religion et au massacre de la Saint Barthélémy qui parsemaient volontiers les discours des Révolutionnaires de i789, n'étaient vues que comme le symbole des horreurs et des abus de l'ancien régime.

La minorité protestante - comme la minorité juive - étaient désormais reconnue civiquement; le fanatisme et le despotisme étaient effacés de la scène de l'histoire : il ne pouvait plus y avoir d'Affaire Calas, ni plus tard il ne pourrait y avoir d'Affaire Dreyfus, c'était du moins la thèse révolutionnaire.

Le mot fanatique qui, au moment de l'affaire et dans son contexte politique religieux et social, devraient désigner seulement les « hérétiques » se retourne, dans les pièces, contre ceux qui l'utilisent, c'est-à-dire les catholiques. Le raccourci historique entre la réalité et sa représentation est tel que fanatique, dans les pièces en vers, rime avec les uns et les autres.

En i76r, pour le camp des catholiques («fanatiques ») de Toulouse, c'est-à-dire les juges qui firent condamner Calas (Clérac pour Chénier; David pour Lemierre) et une partie des habitants de Toulouse, les « fanatiques » étaient les hérétiques:

«Mais je puis concevoir qu'un zèle fanatique

Arme contre son fils la main d'un hérétique » (Clérac, Chénier, A.I s.I)

«Vous ignorez donc tout ce que peut un hérétique (David, Lemierre, A.III,

S.5)

«Mon fils devait le soir faire abjuration »

Disent $-i l s$, « et son père aveugle et fanatique

9. « Ne parlons pas ici de "tolérance"; cette expression dominatrice est une insulte et ne doit plus faire partie du langage d'un peuple éclairé » (Talleyrand, Rapport au nom du comité de Constitution, avril 1791), citê par F. Brunot, Histoire de la langue française des origines à 1goo, TOMғ IX, deuxième partie, Paris, Colin, 1937, p. go2.

Io. Cf. R. Pomeau, La Religion de Voltaire, Paris, Nizet, 1956, p. 321 sq.

II. Charles IX ou l'Ecole des Rois de M.-J. Chénier, représentée pour la première fois au Théâtre de la Nation. 
N'a plus dans son enfant rien vu qu'un catholique:

Et du sang égaré détruisant le saint næud,

Il a tué son fils croyant plaire à son Dieu !» (Madame Calas, Laya, A.II, s.z)

Pour les protestants de Toulouse qui, en I76i, subissaient le fanatisme (Madame Calas, Pierre Calas, Lavaisse) et pour un homme des Lumières, comme le juge La Salle qui lutta de toutes ses forces pour ne pas faire condamner Calas, les « fanatiques »c'étaient d'une part les catholiques qui avaient contribué dans le passé aux massacres des protestants et qui s'apprêtaient à commémorer le bicentenaire du massacre de $1562^{12}$ :

«Au sein de nos remparts de zélés catholiques

Jadis ont immole des milliers dhérétiques

Une fête annuelle est l'affreux monument

Qui retrace à nos yeux ce grand événement;

De ces meurtres sacrés c'est le jour séculaire. » (La Salle, Chénier, A.I, S.I)

«Nous touchons à ce jour!... Déjà des fanatiques

Courent la torche à la main, hurlant d'affreux cantiques » (Lavaisse, Laya,

A.II, s.7)

et d'autre part les catholiques qui, en I76I, n'hésitaient pas à sacrifier Calas :

«Que contre un protestant de pieux catholiques

Cherchent à rallumer leurs torches fanatiques » (Madame Calas, Laya, A.II, s.6)

«Et sans fierté, sans honte, en bute aux traits de rage

D'un peuple fanatique insultant son passage » (Lavaisse, Laya, A.II, S.I)

"D'un peuple furieux contre nous déchaîné

Oui, criait cette foule impie et fanatique » (Madame Calas, Chénier, A.II, s.2)

Quant au mot « fanatisme », qui à l'origine désignait, pour les catholiques, l'hérésie religieuse (celle des calvinistes, mais aussi celle des jansénistes),et qui s'était étendu au cours du XVIII siècle à tout excès religieux, s'assimilant au sens du mot fanatique, il se rapporte dans les pièces presque exclusivement à la religion catholique ${ }^{\mathrm{I} 3}$. Les dictionnaires de langue contemporains ou immédiatement postérieurs à l' Affaire Calas étaient au demeurant prudents et n'entérinaient le glis-

12. Une procession rappelait chaque annee l'expulsion sanglante des huguenots de Toulouse ( 17 mai 1562); l'affaire Calas coïncida avec la préparation du bicentenaire de la « délivrance » que la ville s'apprêtait à célébrer en grandes pompes.

13. Les mots « fanatisme » et « fanatique » ont fait l'objet d'une étude pour la période 1789-1795; $c f$. S. Bianchi, Fanatiquels)/Fanatisme (1789-1795), dans le « Dictionnaire des usages socio-politiques (1770-1815) », fasc.I, INaLF, Paris, Klincksieck, 1985, p. $71-78$. 
sement de sens d'« excès religieux » qu'à mots couverts, sans donner, bien sûr, d'exemples concernant les catholiques ${ }^{14}$. Il fallait s'appeler

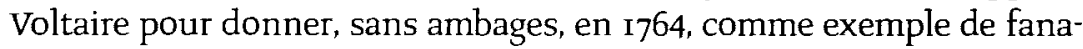
tisme les massacres perpétrés par des catholiques : « le plus détestable exemple de fanatisme est celui des bourgeois de Paris qui coururent assassiner, égorger, jeter par les fenêtres, mettre en pièces, la nuit de la Saint Barthélémy, leurs concitoyens qui n'allaient point à la messe » (Dictionnaire Philosophique, entrée «fanatisme »). Mais le Dictionnaire Philosophique portatif ou la Raison par alphabet n'est pas un dictionnaire de langue et son auteur s'était passé d'autorisation officielle.

Nous n'avons relevé, dans les pièces, qu'un seul exemple de son emploi en tant qu'excès religieux, sans distinction de religions :

"Quoi, des Religions, ce mal inévitable,

Au culte protestant serait-il étranger,

Ou l'esprit d'une secte aurait-il pu changer »/

Non, non; le fanatisme enfante tous les crimes;

Sans égard et sans choix, il frappe ses victimes;

Du sang, de la nature il fait taire la voix... „ (Clérac La Salle, Chénier, A.I, s.I)

Mais les occurrences les plus nombreuses des mots fanatisme et fanatique concernent la justice:

«Le fanatisme condamne les Calas» (David fils, Lemierre, A.III, s.2)

«Envain, vous rappelerais-je ce que la justice, Ihumanite attendent de votre auguste ministère; en vain vous dévoilerais-je les atrocités, les calomnies, les absurdités sans nombre qu'ont enfantées contreles Calas, la haine, le fanatisme,

l'envie.» (David fils, Lemierre, A.III, s.I)

«L Le fanatisme enfante tous les crimes [...

Mais pénétrant aussi dans le Temple des Lois

Souvent, Monsieur /Clérad, souvent, sa terrible puissance

Aux mains des Magistrats fait pencher la balance » (La Salle, Chénier, A.I, s.I)

"L'ceuvre du fanatisme est enfin consommée

Les juges satisfaits; linnocence opprimée » (La Salle, Chénier, A.III, s.4)

«Le fanatisme impur, ce fléau des mortels

Souiller les Tribunaux, les Trônes, les Autels» (Madame Calas, Chẻnier,

$A . V$, s.4)

La pièce de Laya rappelle même le rôle du « Monitoire », cet avis public, pour en appeler à des témoignages qui avait été rédigé de

14. Dictionnaires de langue consultés : Dictionnaire de l'Académie léd. r694; éd.1718; èd. 1740; éd. I762; éd. an VII); Dictionnaire de Trévoux (nouvelle éd. I77I); Dictionnaire critique de la Langue française de Féraud ( $\left.1787^{-17} 88\right)$. 
manière à fournir la preuve d'un complot protestant et à établir que le motif de l'assassinat de Marc-Antoine Calas était religieux :

«D'un sanglant monitoire épouvantant les âmes

Pourquoi du fanatisme attisez-vous les flammes » (La Salle au Capitoul, Laya,

A.III,S.5)

Et surtout ce sont les juges fanatiques qui condamnèrent Calas qui sont dénoncés dans toutes les pièces :

«S'il faut, pour vous fléchir, parler en fanatique

Tel homme est innocent puisqu'il est catholique » (La Salle s'adressant à

Clérac, Chénier, A.I, s.3)

«L culte emporte tout dans son cour fanatique

Et tout homme est jugé qui n'est pas catholique (Le Capitoul là propos de

l'Assesseurl, Laya A.III, s.2)

«les ljugesl fanatiques levaient la tête, prêchaient, sans honte, lintolérance et le

crime.» (Madame Calas, Pujoulx, s.5)

Ces mêmes juges que Voltaire fustigeait dans son Dictionnaire:

Il y a des fanatiques de sang-froid : ce sont les juges qui condamnent à mort ceux qui n'ont d'autre crime que de ne pas penser comme eux [...] (Dict. phil., entrée «Fanatisme »; voir aussi «Certain »).

Au moment où étaient représentées les pièces le thème de la justice était d'actualité puisque la réforme judiciaire, mise en route dès novembre I78g, était encore objet de débats en 179I, avec l'élaboration d'un code pénal pour réglementer l'application des peines.

Sur les cinq auteurs, les trois qui situent l'action de leur pièce pendant le procès, consacrent un acte à l'interrogatoire (Lemierre et Laya, acte III, Chénier, acte II), en plus de nombreuses répliques, ou même scènes où la justice est en cause ${ }^{15}$. Le compte rendu des « Révolutions de Paris » du 26 février-5 mars i7gi, sur la pièce de Lemierre, semblerait confirmer la pertinence du sujet; on reconnaissait à l'auteur le mérite d'avoir « rappele un sujet bien propre à nous attacher au nouvel ordre judiciaire », en « nous faisant frémir à la vue des suites horribles de la jurisprudence de nos ci-devant cours de justice ». De même,

15. Les pièces de Pujoulx et de Willemain d'Abancourt, en un seul acte, se situent au moment de la révision du procès et centrent davantage leur intérêt sur l'intervention de Voltaire. Pujoulx situe l'action de sa pièce au moment de la révision du procès $(1765)$, préférant, affirme-t-il dans sa Préface, « le tableau de la vertu récompensée » à celui du « vice puni ». La condamnation de Calas « fut le triomphe de l'ignorance et du fanatisme; l'areét qui le ressuscita moralement, fut celui de la justice et de la philosophie » (ibid.). 
la note de Willemain d'Abancourt, à la scène 9 de son texte imprimé : « Jaurais pu clouer en cet endroit un morceau de déclamation contre les Anciens Tribunaux, qui peut-être aurait été très applaudi », dans son cynisme, est significative de l'effet produit sur le public. Il ne faudrait pas en conclure pour autant que les contemporains étaient disposés à accepter des caricatures des juges qui condamnèrent Calas. Ainsi, Laya, qui avait voulu montrer dans les juges de Toulouse « les instruments du capitoul qui fait servir le fanatisme à ses projets » (Préface de la pièce imprimée), fut accusé par La Harpe, dans sa Correspondance littéraire, d'avoir « dénaturé le sujet de Calas » en faisant du capitoul «un franc scélérat, suborneur de témoins et digne de la corde ». En revanche, Chénier, malgré ses intentions didactiques et politiques déclarées dans le sous-titre même de sa pièce, lÉcole des Juges, fut apprécié pour avoir présenté dans le juge clérac le fanatisme d'un catholique convaincu de soutenir une juste cause ${ }^{\mathrm{I} 6}$.

Le fanatisme perd son sens général d'excès religieux et finit par désigner la religion catholique, qui, du temps de l'Affaire Calas, pénétrait la machine judiciaire, avec la complicité du pouvoir. Les pièces posent en termes concrets la question des rapports de l'Église avec le pouvoir civil sous l'ancien régime. L'assassinat juridique de Calas apparaît comme l'un des maillons d'une longue chaîne d'ingérences du pouvoir religieux sur le pouvoir civil : l'expression politique maximale de l'intrusion religieuse étant la révocation de l'Édit de Nantes sous le règne de Louis XIV :

«Ce Monarque imposant jusque dans ces faiblesses,

Voulant de son royaume augmenter la splendeur

Sous la religion fit fléchir sa grandeur:

I6. Cf. la Lettre de Palissot (elle aurait dû paraître dans le « Journal de Paris »à la sortie de la pièce en I79I, mais les « objets de la politique » ne lui laissèrent pas d'espace) qui sert de Préface à la tragédie de Chénier : « l...] avoir franchi une difficulté, peutêtre encore plus grande, en faisant un honnête homme du juge qui a le malheur de condamner l'innocence », (PTéface, p. viI), ; cf. aussi « Le Mercure de France » du 23 juillet I79I : « [...] Il y a dans l'ordonnance de cet ouvrage des choses extrêmement adroites, telles que l'idée d'avoir peint dans le caractère du juge un fanatique de bonne foi, sans lui avoir donné un motif particulier pour persécuter la famille des Calas ». Le nom de La Salle, personnage du bon juge que l'on retrouve dans les pièces de Laya, Chénier et Lemierre, est emprunté au conseiller du Parlement qui vota pour l'acquittement de Calas et qui dans son Mémoire accusa la cour de préjugès religieux. Signalons que le rôle du juge La Salle dans la pièce de Chénier était tenu par Talma. En 1794, on jouait encore à Bordeaux Jean Calas, accru d'une nouvelle « suite ». L'Empire des remords ou le Juge de Calas, cf. Paul Courteault, La Révolution et les Théâtres à Bordeaux, Paris, 1926, p. I18 
Et de là vint, Monsieur, cetédit salutaire

Qui livrait l'hérésie au glaive de la loi : [.] 》)

«Ainsi vous exaltez les crimes de vos Princes !I..J

Louis, cet ennemi de toute liberté, L.J

La France qu'appauvrit son luxe despotique

Le vit fouler aux pieds la majesté publique »(Clérac/La Salle, Chénier, A.I, s.I)

En fait, si le poids de la religion a pu peser si fort dans la balance de la justice, c'est le régime sous laquelle celle-ci était exercée qui en est responsable. Il ne s'agit pas seulement d'opposer le fanatisme aux Lumières, à la raison, à la tolérance - le mot fanatisme faisant couple avec obscurantisme, superstition et préjugés - mais d'accuser le régime qui était complice. Le fanatisme et le despotisme vont de pair et la lutte contre le premier est inséparable de celle contre le second :

«Ne peut-on désarmer un cruel fanatisme?

Non; ces grands Tribunaux, rivaux du despotisme,

Affectent son orgueil ainsi que sa fureur » (Louis Calas/ La Salle, Chènier,

A.III,5.4)

«Qu'enfin la liberté succède au despotisme

La douce tolérance, au sanglant fanatisme » (La Salle, Chénier, A.V, s.8)

Dans ce combat, on reconnaît le rôle préparatoire et déterminant de la «philosophie » et de son champion au niveau imaginaire dans la conscience collective, Voltaire. C'est à lui que d'Abancourt et Pujoulx font annoncer, sur la scène, des temps meilleurs ${ }^{17}$ :

«L'odieux fanatisme est encore redoutable

Je voudrais l'éclairer de ce feu respectable

Qu'allume la raison, qu'éteint le préjugé » (Voltaire, d'Abancourt, s.8)

«Mais il viendra l'instant ou la philosophie

Etablissant partout une juste harmonie,

Tirera la raison des fers du préjugé,

17. Dans les deux pièces citées ci-dessus, la présence de Voltaire sur la scène ne doit ni surprendre, ni être considérée comme exceptionnelle. Il s'agit en fait d'un procédé courant dans le théâtre de l'époque révolutionnaire, puisque de nombreuses pièces sont liées à l'événement ou mettent en scène des personnages connus (Rousseau, Mirabeau, Viala, Marat, charlotte Corday, ...). D'ailleurs, dans le cas précis des pièces sur l'Affaire Calas, tous les personnages, ou presque, sont réels et la plupart sont encore vivants. Dans la pièce de Pujoulx, Voltaire fait partie des personnages, bien qu'il ne se fasse reconnaittre qu'à la dernière scène. Avant la grande scène finale de reconnaissance, un premier hommage lui est rendu devant son buste. Dans Ia bienfaisance de Voltaire de W. d'Abancourt, non seulement Voltaire est le personnage principal, mais l'action se déroule à Ferney dans son château. 
Et nos neveux verront l'homme libre et vengé;

Ils verront tôt ou tard l'odieux despotisme

Rentrer dans le néant avec le fanatisme » (Voltaire, d'Abancourt, s.g)

"J'ai vu, il y a trois ans, Inous sommes en 1765 l les habitants de Toulouse attendre avec la même impatience l'arrêt qui assassina Jean Calas. Mais quelle différence ! le fanatisme les embrasait; et peut-être que la philosophie éclairera les nouveaux juges de cette famille »(Voltaire, Pujoulx, s.I)

«Cest à cet homme célèbre, aussi grand par sa bienfaisance que par son génie, que vous devez l'arrêt qui proclame l'innocence d'une victime du fanatisme » (lavocat de Mme Calas, Pujoulx, S.I2)

Le compte rendu du « Moniteur » du 3 juin I79i à La Bienfaisance de Voltaire de Pujoulx explicitait le message et en confirmait la réception : « [...] Voltaire, qui dans tout le cours de l'ouvrage, a parlé avec chaleur contre la persécution, l'ignorance, la superstition et le despotisme s'échauffe ici davantage lon vient d'apporter la nouvelle de la réhabilitationl, et, perçant la nuit des temps, il présage que bientôt la philosophie couvrira l'Europe de ses lumières, que le fanatisme sera détruit, qu'un nouvel ordre de choses rendra les hommes libres et heureux, et que son nom sera placé parmi ceux des écrivains courageux qui auront produit une si grande Révolution. Cet ouvrage [...] a été très applaudi ».La vision utopique de Voltaire devient ainsi histoire et cautionne la Révolution.

C'est pourquoi, dans les pièces, le « peuple [fanatique] » de Toulouse n'est pas exclus de la régénération collective, même si on n'hésite pas à rappeler son rôle, au demeurant prouvé par les faits, dans la condamnation de Calas $^{18}$ :

Le sentiment public s'est trop manifesté:

De la prévention vous connaissez l'empire.» (Le religieux, Chénier, A.III, s.3)

La voix du peuple enfin l'accuse et !... » (Le Capitoul. Laya, A.II, s.6)

N'avons-nous pas entendu dire parmi le peuple que Calas avait assassiné son

fils ? »[..] «Les témoins et les preuves ne nous manqueront pas; le peuple va nous

en foumir. Au reste, sommes nous les seuls qui sentions la nécessité d'anéantir cette race impie? » (2 Capitoul, Lemierre, A.II, s. g)

«Un peuple qui dans son zèle aveugle; ne connait aucun frein; à qui le fanatisme fait tout croire, parce que le fanatisme lui rend tout possible; qui employant contre une secte particulière la violence et l'outrage, croit obéir à Dieu et venger son injure; un peuple que des prêtres sacrilèges trompent et soulèvent par des cérémonies religieuses : ce peuple doit-il être pour un juge

18. Cf. D. Bien, L'Affaire Calas, en particulier chap. VI, « Calas en procès : l'opinion populaire $»$. 
l'organe de Dieu même? » (La Salle, Lemierre, A.III, s.5)

Mais on nie sa responsabilité, le « peuple » étant lui-même une victime du fanatisme :

«le peuple qu'on égare » (Le religieux, Chénier, A.I, s.2)

«le peuple égaré » (La Salle, Chénier, A.I, s.3) (Lavaisse, Laya, A.II, s.I)

«Voilà bien le peuple, lorsque le fanatisme l'égare! » (Caseing, Lemierre A.II,

s.7)

«Pourquoi du fanatisme attisez-vous les flammes?

Sur ce peuple à l'erreur se laissant emporter

Si prompt à la saisir, si lent à la quitter

Et dont la vertu même est un excés à craindre » (La Salle, Laya, A.III, s.5)

L'accusation de fanatisme ne concerne donc pas le peuple, mais ceux qui le manipulent. Le fanatisme renvoie en fait à une catégorie beaucoup plus vaste qui englobe tous les ennemis du peuple, les diviseurs du peuple, tous ceux qu'il faut combattre pour que le peuple reste uni. Le peuple de la Révolution ne peut être qu'unanime.

C'est dans cette optique qu'il faut interpréter l'absence absolue, dans les cinq pièces de i790-9I, du mot " populace » largement utilisé par Voltaire en 1763 (v. par exemple, Traité sur la Tolérance ${ }^{19}$, chap.I : « populace fanatique », « vile populace », [...] ou encore par Mercier en I77I, « populace effrénée », v. citation ci-dessus de l'An 2440) pour désigner les catholiques (fanatiques) de Toulouse.

A la limite, il s'agit moins de dénoncer les catholiques fanatiques de Toulouse, dont le fanatisme était conjoncturel et appartient desormais au passé, que d'être attentif à ceux qui continuent à égarer le peuple. Dans le contexte du fameux Serment constitutionnel qui coïncide avec la période où sont représentées les pièces. le catholicisme n'est plus seulement un comportement religieux mais un choix politique, qui se précise encore après la condamnation de la Constitution civile du clergé par le pape Pie VI en avril i7gr. La connotation politique du mot fanatisme devient toujours plus liée à la Révolution, les fanatiques finissant par désigner les catholiques qui n'adhèrent pas à la Révolution. Une pièce jouée en juin I791 , La Ligue des Fanatiques et des Tyrans, confirme le glissement de sens : les (catholiques) fanatiques, au même titre que les tyrans et les despotes sont les ennemis de la Révolution $^{20}$. En l'an II, l'amalgame entre fanatisme (catholicisme solidaire de la monarchiel et contre-tévolution sera accompli. A partir de ce moment, le mot fanatisme, qui tend à se politiser toujours davantage

19. Cf. Voltaire, Traité sur la tolérance, Paris, Flammarion, 1989. 
retrouve en quelque sorte son sens originel d'excès. Non plus religieux au sens strict, mais de zèle en toute croyance, il finira même par caractériser, par un incroyable retournement de sens, le discours révolutionnaire ${ }^{2 \mathrm{I}}$.

Désormais, la question religieuse et son corollaire l'anticléricalisme, définissent, et pour plus d'un siècle, les camps politiques. A partir du Second Empire le mot cléricalisme prendra la relève du mot fanatisme dans le sens qui nous intéresse ici : l'« Écrasons l'Infâme »de Voltaire fera place au « cléricalisme voilà l'ennemi » de Gambetta ${ }^{22}$.

A la leçon politique des pièces s'ajoute la leçon civique et morale: l'aspect privé, affectif, de l'Affaire Calas n'est pas un des moindres. La représentation d'une famille modèle (un père attentif, une mère vertueuse, des enfants unis),telle qu'elle est d'ailleurs decrite par Voltaire dans son "Histoire abrégée de la mort de Jean Calas" (premier chapitre du Traité surla Tolérance ${ }^{23}$, montre, d'une part, l'importance de la famille dans l'échelle des valeurs révolutionnaires et, d'autre part, la validité du drame bourgeois, en passe de devenir mélodrame, dans le théâtre de la Révolution.

L'exemple le plus évident, à la fois de la persistance du genre et de sa tendance mélodramatique, est celui du drame de Lemierre, Calas oule fanatisme, où les didascalies du premier et du quatrième acte qui décrivent deux scènes familiales des Calas lavant et après la mort de MarcAntoine) évoquent un «tableau », au sens où l'entendait Diderot ${ }^{24}$.

20. «Tragédie nationale » de Ch.-Ph. Ronsin, en trois actes et en vers, representée au Théâtre Molière. Signalons encore que le théâtre se fait aussi l'écho d'une autre forme d'anticléricalisme, moins moderne, dans la tradition médiévale, qui concerne l'amoralité du clergé, mais aussi le thème plus actuel des voux forcés : La Journée du Vatican ou le Mariage du Pape, Les fourberies monacales, Le Mari directeur ou le déménagement du couvent, les Victimes cloitrées, [... On joue aussi des pièces sur le thème du Serment constitutionnel, comme par exemple Le Serment du vicaire.

2I. F. Brunot, Histoire de la langue, томe IX, op. cit., p. 626.

22. Nous citons un court extrait de la définition de clérical du Larousse du XIX $:$ « [...] Ce n'est donc point contre la religion que nous avons voulu diriger nos attaques et nos critiques; ce n'est pas contre la partie saine, la partie éclairée du clergé, c'est uniquement contre ceux qui veulent tourner les forces vivaces de la religion contre la marche progressive des idées $(. .)$.$» .$

23. Le texte imprimé de Laya est précédé d'une « Préface historique sur Jean Calas » qui est reprise du Traité sur la Tolérance ; de même le drame de Lemierre est précédé d'une « Histoire abrégée de la mort de Jean Calas tirée des ceuvres de Voltaire » de 23 pages.

24. Didascalie du Premier acte : « Le théâtre représente un salon. Il est éclairé. A droite des spectateurs, on voit la porte de la salle à manger, et c'est de ce côté que madame Calas s'occupe à filer de la soie, tandis que ses deux filles travaillent, l'une à broder 
Chénier, le tenant de la tragédie nationale, renchérit sur le mélodrame et symbolise la réprobation du ciel contre la condamnation de Calas par un violent orage ${ }^{25}$ :

\section{Didascalie de l'Acte III :}

«La scène est dans une place où la prison est située. Un orage se prépare durant les premières scènes, et les éclairs se pressent avec rapidité ». À la scène 5 Idialogue entre Madame Calas et le « méchant » juge Clérac), " l'orage s'accroît durant toute la scene ».

Encore, dans les pièces de Laya et de Chénier, définies tragédies peut-être parce qu'elles sont en vers ${ }^{26}$ - l'intervention de Voltaire, sauveur inespéré de la famille Calas, peut prendre des allures romanesques:

«J'apprends que ce grand homme, honneur de son pays

Et qui du fanatisme intrépide adversaire,

Eteindra ces bûchers qui dépeuplent la terre;

De Fermey dans nos murs arrivé dans ce jour.

Y va pour quelque temps établir son séjour » (Laya, ActeV, scène dernière)

une robe, l'autre de la mousseline. À gauche, se trouve une table sur laquelle MarcAntoine écrit une lettre que lui dicte son père, qui est debout près de lui. Au fond, une porte qui mène à l'escalier du magasin. »

La même scène de famille dans le cachot, huit jours après le jugement (Quatrième acte) :

«Calas, les fers aux pieds, et assis sur une chaise de bois, dort d'un sommeil tranquille, Madame Calas, plongée dans la plus vive douleur, est dans un fauteuil, à quelque distance de son mari; Jeanne se tient debout à côté d'elle. Rose et AnneRose sont aux pieds de leur père, et se penchent, l'une sur son sein, l'autre sur ses genoux. Elles versent des larmes. Pierre s'appuie sur une table dans une attitude douloureuse. Lavaisse paraît d'abord assis dans un coin, mais bientot il se lève et s'approche de Calas. »

25. Mais il semblerait que l'orage n'ait pas eu l'effet escompté : « On a blâmé une tempête qui accompagne le troisieme acte, et qui a paru durer beaucoup trop longtemps sans produire l'effet que l'auteur devait en attendre. De pareils moyens ne peuvent que nuire quand ils ne servent pas infiniment; l... le bruit du tonnerre n'avait d'autre effet que de couvrir la voix des spectateurs » 《 Le Moniteur », 24 juillet I79I).

26. «Le Moniteur », annonce le Jean Calas de Chénier comme tragédie, mais Quérard [France littéraire] indique le titre de Jean Calas ou lEcole des juges, drame en cinq actes et en vers pour une édition de 1792 chez Moutard. L'édition de I793, toujours chez Moutard, porte Jean Calas, tragédie. L'édition des ceuvres completes indique «drame ». Cf. aussi la notice d'Arnault : « A ne considérer que la condition des personnages, Calas ne serait qu'un drame; à ne considérer que le fait qui s'y développe et la catastrophe qui le dénoue, c'est une véritable tragédie ». Sur le problème tragédie /drame, v. aussi la Préface de Chénier à son Fénelon (Réflexions sur Fénelon, T. IV des Oeuures, 1797). 
"Il est près des Monts Helvétiques

Un illustre Vieillard, féau des fanatiques » (La Salle, Chénier, A.V, s.2)

«Si je les défendrai ! je le veux, je le dois

-. $\quad$ legénie

Va s'armer, va tonner sur le sénat impie;

Va dévoiler la trame où le juste est frappé »(Voltaire, Laya, A.V, scène

dernière)

Nous serions tenté de conclure qu'au fond c'est justement le drame, ce genre défini et aimé par les philosophes, qui fournit la clé de la modalisation théâtrale et révolutionnaire de l'Affaire Calas : les 'menaces' qui ont pesé sur la famille Calas représentent à la fois l'image et l'effet réel des dangers de l'ancien régime.

D'ailleurs, est-ce que ce ne fut pas par le biais du theâtre que Voltaire entama publiquement sa lutte contre le fanatisme en faisant représenter Mahomet? mais l'Affaire Calas n'avait pas été pas une invention théâtrale.

En pleine déchristianisation, on décrètera d'élever, à Toulouse, en hommage à Calas, une colonne « construite du marbre arraché au fanatisme dans les églises supprimées ». La colonne ne fut pas construite, nous en rappellerons cependant le décret :

Citoyens,

La nature, la loi, la justice, la vérité, ont été outragées par la sanglante condamnation de Calas; le fanatisme et l'erreur siégeaient avec ses juges et leur ont dicté son arrêt de mort. Vingt mille spectateurs ont frémi en voyant ce vieillard irréprochable sur l'échafaud. Ceux qui demandaient son supplice, pour venger la religion, ont répandu des larmes sur son bûcher; et l'Europe entière, dont ce procès célèbre avait fixé les regards, a été indignée.

La réhabilitation de sa mémoire sous le despotisme ne pouvait être une reparation suffisante. Il n'appartenait qu'aux représentants d'un peuple libre, juste et éclairé du flambeau de la raison, d'attester, par un monument public, à la postérité la plus éloignée, l'innocence du plus malheureux des pères.

Vous avez rendu hommage à la nature et à la plus douce des vertus, au nom de tous les hommes libres, en décrétant solennellement, le 29 brumaire dernier (ig novembre i793), qu'il serait élevé, aux dépens de la République, sur la place où le fanatisme a fait périr Calas, une colonne en marbre, sur laquelle serait gravée l'inscription suivante :

La Convention nationale,

à la nature,

à l'amour paternel 
à Calas, victime du fanatisme.

Vous avez rendu hommage à la raison, en décrétant que cette colonne serait construite du marbre arraché au fanatisme dans les églises supprimées $\left[. . .\left.\right|^{27}\right.$.

\section{Université de Bari}

Italie

27. « Rapport et projet de decret sur la proposition d'indemniser les enfants de Jean Calas, de la ruine que son procès leur a occasionnée, aux dépens de qui il appartiendra; présentés, au nom du Comité de Législation, par F.S. Bezard, député par le département de l'Oise à la Convention nationale. » (Séance du 23 pluviôse) (mars 1794). 